\title{
openheart Specialist intervention is associated with improved patient outcomes in patients with decompensated heart failure: evaluation of the impact of a multidisciplinary inpatient heart failure team
}

\author{
Jayne Masters, ${ }^{1}$ Geraint Morton, ${ }^{2}$ Isabel Anton, ${ }^{3}$ Jane Szymanski, ${ }^{1}$ \\ Elizabeth Greenwood, ${ }^{1}$ Joanna Grogono, ${ }^{4}$ Andrew S Flett, ${ }^{1}$ John G F Cleland, ${ }^{5}$ \\ Peter J Cowburn ${ }^{1}$
}

To cite: Masters J, Morton G, Anton I, et al. Specialist intervention is associated with improved patient outcomes in patients with decompensated heart failure: evaluation of the impact of a multidisciplinary inpatient heart failure team. Open Heart 2017;1:e000547. doi:10.1136/ openhrt-2016-000547

Received 6 0ctober 2016 Revised 5 December 2016 Accepted 10 January 2017

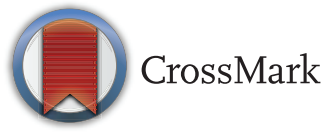

'Department of Cardiology, University Hospital

Southampton, Southampton, UK 2Department of Cardiology,

Portsmouth Hospitals NHS Trust, Portsmouth, UK

${ }^{3}$ Facultad de Ciencias de la Salud, Universidad San Jorge, Zaragoza, Spain

${ }^{4}$ Department of Cardiology, John Radcliffe Hospital, Oxford, UK ${ }^{5}$ Royal Brompton and Harefield Hospitals, National Heart and Lung Institute, Imperial College London, London, UK

Correspondence to Dr Geraint Morton; geraintmorton@gmail.com

\section{ABSTRACT}

Objective The study aimed to evaluate the impact of a multidisciplinary inpatient heart failure team (HFT) on treatment, hospital readmissions and mortality of patients with decompensated heart failure (HF).

Methods A retrospective service evaluation was undertaken in a UK tertiary centre university hospital comparing 196 patients admitted with HF in the 6 months prior to the introduction of the HFT (pre-HFT) with all 211 patients seen by the HFT (post-HFT) during its first operational year.

Results There were no significant differences in patient baseline characteristics between the groups. Inpatient mortality ( $22 \%$ pre-HFT vs $6 \%$ post-HFT; $p<0.0001)$ and 1 -year mortality (43\% pre-HFT vs $27 \%$ post-HFT; $p=0.001$ ) were significantly lower in the post-HFT cohort Post-HFT patients were significantly more likely to be discharged on loop diuretics ( $84 \%$ vs $98 \% ; p=<0.0001$ ), ACE inhibitors ( $65 \%$ vs $76 \% ; p=0.02$ ), ACE inhibitors and/ or angiotensin receptor blockers ( $83 \%$ vs $91 \% ; p=0.02$ ), and mineralocorticoid receptor antagonists (44\% vs $68 \%$; $\mathrm{p}<0.0001)$ pre-HFT versus post-HFT, respectively. There was no difference in discharge prescription rates of betablockers ( $59 \%$ pre-HFT vs $63 \%$ post-HFT; $p=0.45)$. The mean length of stay $(17 \pm 19$ days pre-HFT vs $19 \pm 18$ days post-HFT; $p=0.06)$, 1-year all-cause readmission rates ( $46 \%$ pre-HFT vs $47 \%$ post-HFT; $\mathrm{p}=0.82$ ) and HF readmission rates ( $28 \%$ pre-HFT vs $20 \%$ post-HFT; $p=0.09$ ) were not different between the groups. Conclusions The introduction of a specialist inpatient HFT was associated with improved patient outcome. Inpatient and 1-year mortality were significantly reduced. Improved use of evidence-based drug therapies, more intensive diuretic use and multidisciplinary care may contribute to these differences in outcome.

\section{INTRODUCTION}

Despite major advances in medical and device therapy, the prognosis of patients hospitalised with heart failure (HF) remains

\section{KEY MESSAGES}

What is already known about this subject?

- Patients hospitalised with heart failure represent a large and growing healthcare burden. These patients have a poor prognosis with very high inpatient and early postdischarge mortality. Outpatient specialist multidisciplinary care improves outcome for these patients. However, very little is known regarding the impact of specialist teams treating inpatients with decompensated heart failure.

What does this study add?

- Introduction of a multidisciplinary team targeting inpatients with decompensated heart failure was associated with significant reductions in inpatient and 1-year mortality.

How might this impact on clinical practice?

- Recent UK National Institute for Health and Care Excellence guidelines for acute heart failure recommend early and continuing input of a specialist heart failure team for all patients admitted to hospital with heart failure. Our work strongly supports these guidelines as outcomes were significantly improved in patients managed by the multidisciplinary heart failure team. Hospitals admitting patients with heart failure who do not have a dedicated multidisciplinary heart failure team should consider introducing one.

poor. In the latest UK National Heart Failure Audit (2013/2014), 9.5\% of such patients died during their hospital stay. For those who survived to discharge, the 5-year mortality for patients admitted between 2009 and 2014 was $45.5 \%$, with a median follow-up period 
of only 473 days. ${ }^{1}$ Patients who were not managed and followed up by cardiologists were significantly more likely to die than those who were, even after adjustment for confounders. ${ }^{1}$ The financial burden is also significant, with $\mathrm{HF}$ estimated to account for $2 \%$ of the total National Health Service (NHS) expenditure and 5\% of all emergency hospital admissions in the UK. ${ }^{2}$ Furthermore, HF admissions are projected to increase by $50 \%$ over the next 25 years, mainly due to an ageing population. ${ }^{34}$

$\mathrm{HF}$ is a complex syndrome and causes multisystem morbidity, psychological ill-health and social problems. Because HF is predominantly a disease affecting older people, there are also frequently adverse interactions between HF and pre-existing comorbidities. Consequently, the management of HF needs to be multifaceted to reflect this. The importance of specialist multidisciplinary care for patients with HF is reflected in national and international guidelines and is strongly recommended by National Institute for Health and Care Excellence, the European Society of Cardiology (1A recommendation) and the American Heart Association/ American Stroke Association (1B recommendation). ${ }^{256}$ Multidisciplinary care in the outpatient setting improves patient well-being, reduces hospital admissions and improves outcome. ${ }^{78}$ There are, however, few data available on the impact of specialist teams treating inpatients with decompensated HF. We now report on the impact of introducing a specialist heart failure team (HFT) in a university hospital in the UK. The team was launched on a background of a poor performance in a National Health Care Commission Audit of Heart Failure Management. The aim of the HFT was to provide equal access to specialist care wherever the patient presented within the hospital.

\section{METHODS}

This is a single-centre, retrospective, service evaluation performed at University Hospital Southampton NHS Trust, UK, after an HFT was established. The team comprised two specialist HF nurses, a part-time pharmacist and a clinical fellow, and was led by a consultant cardiologist with a specialist interest in HF. The HFT reviewed and optimised the care of all patients referred with a primary admitting diagnosis of HF regardless of patients' location in the hospital or the speciality of the responsible team. There were no specific referral criteria, but we encouraged referral of all patients with a primary diagnosis of HF. HFT input included medication management, regular reviews throughout admission and early outpatient follow-up ( $<2$ weeks postdischarge) where appropriate. Selected appropriate patients were considered for transfer to a cardiology bed for more intensive treatment.

A detailed, systematic, unblinded case note review was conducted on all patients seen by the HFT during its first year of operation (post-HFT) and all patients hospitalised with a primary diagnosis of HF in the 6 months immediately prior to the HFT commencing work (preHFT). A 6-month period was used to include a similar number of patients preintroduction and postintroduction of the HFT.

The data collected included medications on admission and on discharge, length of hospital stay, therapies received, inpatient deaths, follow-up plans and readmissions. One-year mortality was determined from the case notes, hospital computer records and by phoning the general practitioner surgery if required. Comparisons were then made between the pre-HFT and post-HFT groups. Formal ethical approval was not required; however, the study was registered with the Trust's Clinical Effectiveness Department.

\section{STATISTICAL ANALYSIS}

IBM SPSS Statistics (version 20.0) software was used for all statistical analyses. Data are presented as mean $\pm \mathrm{SD}$, except where stated. For continuous variables Shapiro-Wilk analyses checked normality of the underlying distribution. Having determined that non-parametric tests were required, the two groups were compared using the Mann-Whitney U test. The ${ }^{2}$ test was used for comparison of binomial data. Significance was determined if two-sided $\mathrm{p}$ values were $<0.05$. Kaplan-Meier curves were derived to compare mortality between the groups.

In-patient data were complete for both cohorts. Follow-up data were complete in terms of mortality in the post-HFT cohort but was incomplete on three patients in the pre-HFT cohort. These three patients were considered to be alive and not readmitted to hospital for the sake of analysis.

\section{RESULTS \\ Patient population}

During its first year of operation, the HFT reviewed 211 patients (post-HFT) with a mean age of $72.0 \pm 13.3$ years. In the preceding 6 months, 215 patients were coded as having a primary diagnosis of HF; case note review confirmed that 196 patients had been correctly coded. The mean age of these 196 patients (pre-HFT) was $74.0 \pm 13.2$ years. This trend towards slightly younger patients in the post-HFT group did not reach statistical significance $(p=0.08)$. The baseline characteristics of the two cohorts are detailed in table 1.

There were no significant differences in important clinical characteristics between the two cohorts; there were similar proportions of patients with a history of ischaemic heart disease, atrial fibrillation, hypertension and diabetes and of female patients. Renal function, serum sodium, heart rate and systolic blood pressure on admission were also similar between the two groups. There was a non-significant trend towards slightly younger patients with more severe LV dysfunction in the post-HFT cohort. Echocardiography was performed during the index admission in $82 \%$ of the pre-HFT and $79 \%$ of the post-HFT $(\mathrm{p}=0.47)$ groups. 
Heart failure and cardiomyopathies

Table 1 Baseline characteristics

\begin{tabular}{llll}
\hline & Pre-HFT & Post-HFT & p Value \\
\hline Age & $74.0 \pm 13.2$ years & $72.0 \pm 13.3$ years & 0.08 \\
\hline Female gender & $70(36 \%)$ & $85(40 \%)$ & 0.34 \\
\hline IHD & $103(51 \%)$ & $107(53 \%)$ & 0.67 \\
\hline Prior MI & $72(35 \%)$ & $69(34 \%)$ & 0.82 \\
\hline Diabetes & $54(28 \%)$ & $55(26 \%)$ & 0.74 \\
\hline Heart rate & $87 \pm 21 \mathrm{bpm}$ & $89 \pm 26 \mathrm{bpm}$ & 0.77 \\
\hline Systolic BP & $126 \pm 28 \mathrm{mmHg}$ & 0.62 \\
\hline AF & $84(43 \%)$ & $126 \pm 25 \mathrm{mmHg}$ & 0.27 \\
\hline QRS duration & $117 \pm 37 \mathrm{~ms}$ & $102(48 \%)$ & 0.50 \\
\hline Moderate/severe LVSD & $137(70 \%)$ & $116 \pm 44 \mathrm{~ms}$ & 0.06 \\
\hline Preserved LV & $31(15 \%)$ & $165(78 \%)$ & 0.5 \\
\hline Sodium & $135 \pm 6 \mathrm{mmoL} / \mathrm{L}$ & $30(14 \%)$ & 0.53 \\
\hline Urea & $12 \pm 8 \mathrm{mmoL} / \mathrm{L}$ & $135 \pm 6 \mathrm{mmoL} / \mathrm{L}$ & 0.11 \\
\hline eGFR & $48 \pm 23 \mathrm{~mL} / \mathrm{min} / 1.73 \mathrm{~m}^{2}$ & $12 \pm 12 \mathrm{mmoL} / \mathrm{L}$ & 0.27 \\
\hline Haemoglobin & $122 \pm 22 \mathrm{~g} / \mathrm{L}$ & $51 \pm 22 \mathrm{~mL} / \mathrm{min} / 1.73 \mathrm{~m}^{2}$ & \\
\hline
\end{tabular}

AF, atrial fibrillation/flutter; BP, blood pressure; bpm, beats per minute; eGFR, estimated glomerular filtration rate; HFT, heart failure team; IHD, ischaemic heart disease; LV, left ventricle; LVSD, left ventricular systolic dysfunction; MI, myocardial infarction.

\section{Mortality}

Inpatient and 1-year mortality were significantly reduced in the post-HFT cohort. In the pre-HFT group, 44 out of 196 patients died as an inpatient (22\% inpatient mortality), whereas 13 out of 211 patients in the post-HFT group died (6\% inpatient mortality) $(\mathrm{p}<0.0001)$. At 1-year postadmission, 84 out of 196 patients $(43 \%)$ had died in the pre-HFT cohort, whereas 57 out of 211 patients (27\%) had died in the post-HFT cohort ( $\mathrm{p}=0.001)$ (figures 1 and 2).

\section{Pharmacotherapy}

On admission there were no differences in the prescription rates of ACE inhibitors (ACE-I), angiotensin receptor blockers (ARB), ACE-I and/or ARB, beta-blockers, mineralocorticoid receptor antagonists (MRAs) or loop diuretics between the pre-HFT and post-HFT groups, respectively (table 2). There was a significantly higher proportion of patients taking thiazides on admission in the post-HFT group.

On discharge, the prescription rates of both medications with known prognostic benefit (ACE-I, ACE-I and/or ARB, and MRA) and medications with a symptomatic benefit (loop and thiazide diuretics) were significantly higher in patients in the post-HFT cohort (table 3). During their hospital stay, post-HFT patients were more likely to receive intravenous loop diuretics, more likely to be discharged

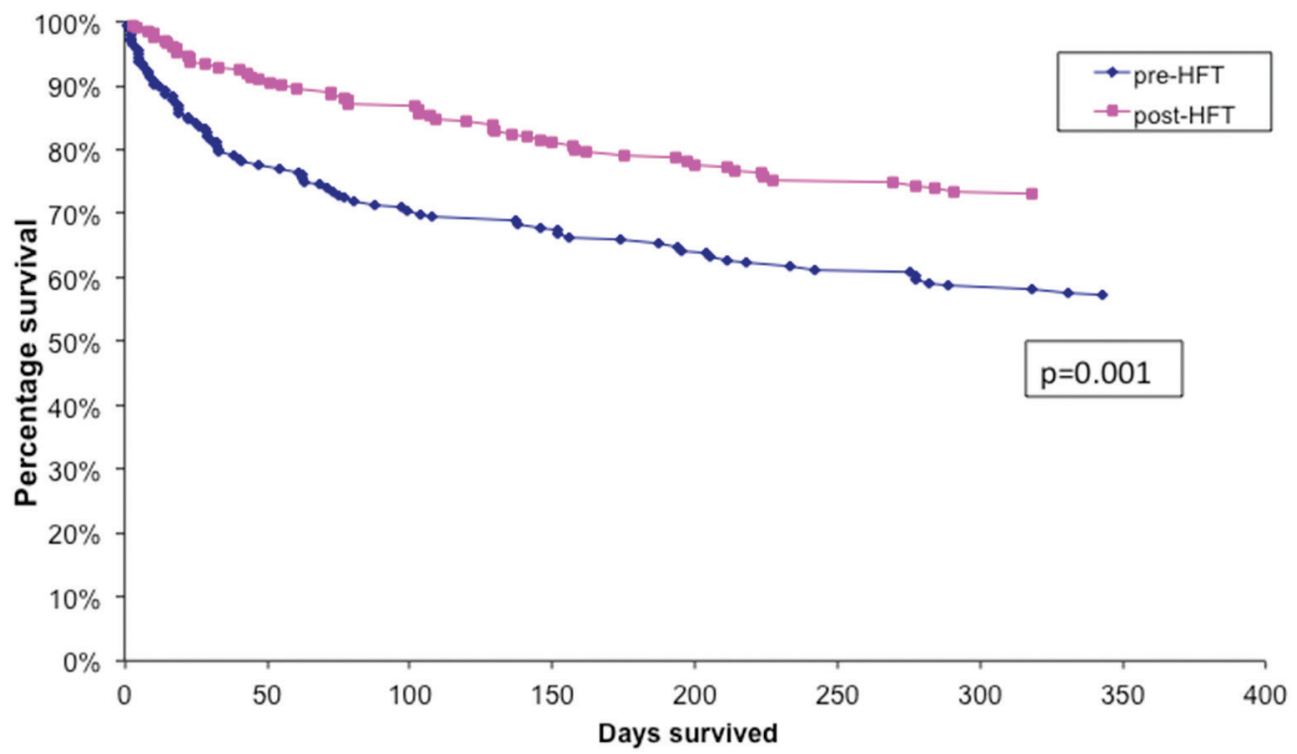

Figure 1 Kaplan-Meier curve showing survival after admission to hospital in the pre-HFT and post-HFT cohorts. Survival was significantly higher in the post-HFT cohort. HFT, heart failure team. 


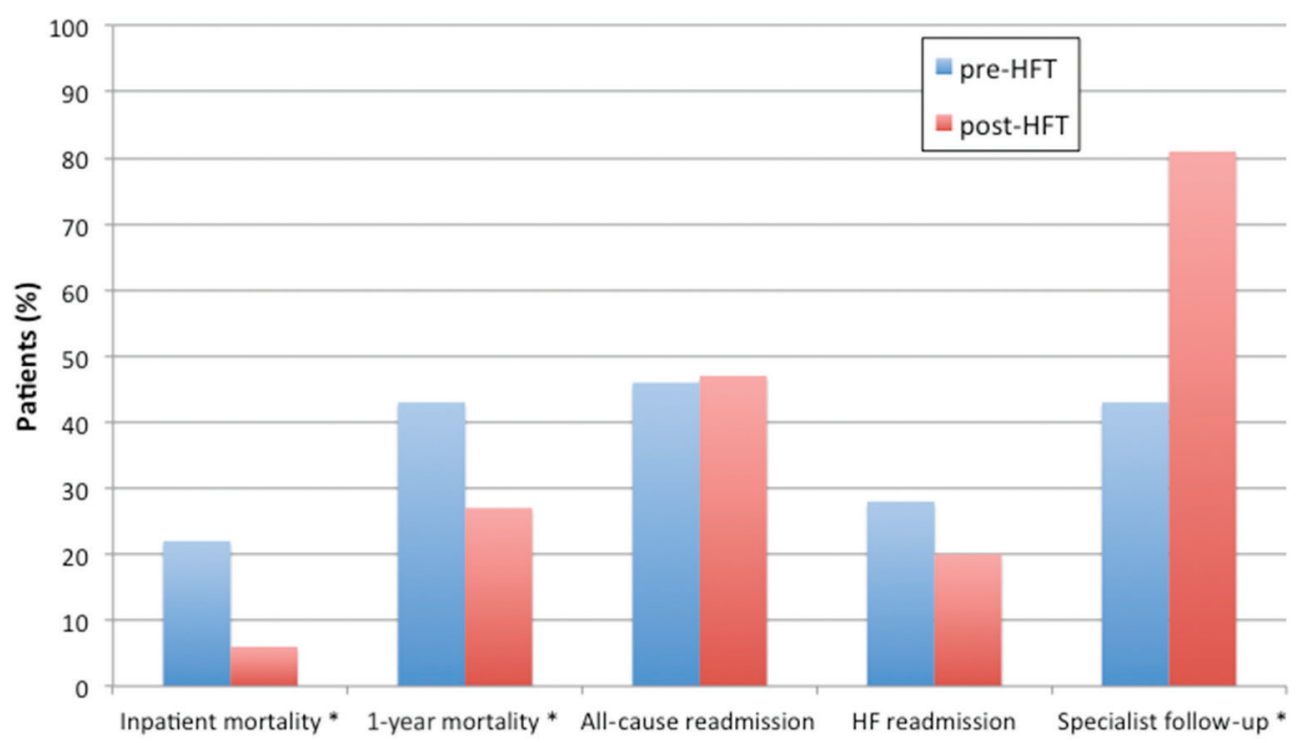

Figure 2 Mortality, 1-year all-cause and HF readmission and specialist follow-up rates in the pre-HFT and post-HFT cohorts. *Denotes statistically significant difference between pre-HFT and post-HFT. HFT, heart failure team.

on a diuretic and were discharged on higher doses of loop diuretic. Intravenous loop diuretic was used in $76 \%$ of pre-HFT versus $88 \%$ of post-HFT patients $(\mathrm{p}<0.002)$. Eighty-four per cent of the pre-HFT patients were discharged on a loop diuretic (70\% furosemide and $14 \%$ bumetanide) compared with $98 \%$ of post-HFT patients ( $54 \%$ furosemide and $45 \%$ bumetanide) $(\mathrm{p}<0.0001)$.

Post-HFT patients received higher doses of oral diuretics on discharge: the mean bumetanide equivalent dose (bumetanide $1 \mathrm{mg}$ = furosemide $40 \mathrm{mg}$ ) was $2.4 \mathrm{mg}$ in the post-HFT cohort versus $1.6 \mathrm{mg}$ in the pre-HFT cohort $(\mathrm{p}<0.001)$. Discharge prescription rates of thiazide diuretics were also more frequent in the post-HFT patients: $5 \%$ pre-HFT versus $17 \%$ post-HFT $(\mathrm{p}<0.001)$.

\section{Advanced HF therapies}

No significant differences in the use of advanced therapies were observed between the pre-HFT and post-HFT cohorts. This includes the use of intravenous inotropes (22\% vs $19 \% ; \mathrm{p}=0.39)$, intra-aortic balloon pump (5\% vs $2 \% ; \mathrm{p}=0.15$ ) and cardiac resynchronisation therapy $(0.5 \%$ vs $2 \% ; \mathrm{p}=0.12)$ pre-HFT and post-HFT, respectively.

\begin{tabular}{llll} 
Table 2 & Admission medications & \\
\hline Medication & Pre-HFT & Post-HFT & p Value \\
\hline Loop diuretic & $60 \%$ & $62 \%$ & 0.59 \\
\hline ACE inhibitor & $48 \%$ & $46 \%$ & 0.8 \\
\hline ARB & $15 \%$ & $15 \%$ & 0.95 \\
ACE-I and/or ARB & $62 \%$ & $61 \%$ & 0.82 \\
\hline Beta-blocker & $37 \%$ & $40 \%$ & 0.56 \\
MRA & $19 \%$ & $23 \%$ & 0.35 \\
\hline Thiazide & $5 \%$ & $11 \%$ & 0.01 \\
\hline
\end{tabular}

ARB, angiotensin receptor blockers; HFT, heart failure team; MRA, mineralocorticoid receptor antagonists.
Furthermore there were no significant differences in the number of patients receiving ventilatory support, including both non-invasive ventilation ( $16 \%$ vs $13 \% ; \mathrm{p}=0.4)$ and intubation and ventilation on intensive care $(14 \%$ vs $8 \%$; $\mathrm{p}=0.06)$, pre-HFT and post-HFT, respectively.

\section{Length of stay, readmissions and specialist follow-up}

The mean length of stay was similar at $17 \pm 19$ days pre-HFT and $19 \pm 18$ days post-HFT $(\mathrm{p}=0.06)$ (figure 2$)$. Of those patients successfully discharged from hospital in the pre-HFT cohort, 67 out of 145 patients (46\%) were readmitted to the hospital as an emergency in the subsequent year, whereas 93 out of 196 (47\%) were admitted from the post-HFT cohort $(\mathrm{p}=0.82)$. Of the pre-HFT readmissions, 40 out of $144(28 \%)$ were due to HF compared with 39 out of $196(20 \%)$ of the post-HFT readmissions $(\mathrm{p}=0.09)$.

Of the pre-HFT cohort, $43 \%$ had specialist follow-up (defined as cardiology, HFT or primary care HF specialist nurse follow-up), whereas $81 \%$ had specialist follow-up in the post-HFT group $(\mathrm{p}<0.0001)$.

\begin{tabular}{|c|c|c|c|}
\hline Medication & Pre-HFT & Post-HFT & p Value \\
\hline Loop diuretic & $85 \%$ & $98 \%$ & $<0.0001$ \\
\hline ACE inhibitor & $65 \%$ & $76 \%$ & 0.02 \\
\hline ARB & $18 \%$ & $16 \%$ & 0.67 \\
\hline ACE-I and/or ARB & $83 \%$ & $91 \%$ & 0.02 \\
\hline Beta-blocker & $59 \%$ & $63 \%$ & 0.45 \\
\hline MRA & $44 \%$ & $68 \%$ & $<0.0001$ \\
\hline Thiazide & $5 \%$ & $17 \%$ & 0.001 \\
\hline
\end{tabular}

ARB, angiotensin receptor blockers; HFT, heart failure team; MRA, mineralocorticoid receptor antagonists. 


\section{DISCUSSION}

After the introduction of an inpatient HFT, the outcome of patients admitted with decompensated HF was much better than the outcome in a very similar cohort admitted in the preceding 6 months. Patients managed by the HFT had significantly lower inpatient mortality and this benefit was maintained at 1-year postadmission. This might reflect improvements in the rates of prescribing of evidence-based medications and the rates of specialist follow-up but did not appear to be due to the use of more advanced therapies.

$\mathrm{HF}$ is an important and growing socioeconomic problem. Although specialist multidisciplinary care has been shown to improve outcomes for HF patients, ${ }^{8}$ the vast majority of published data relate to outpatient programmes, typically beginning at the time of patient discharge. Given the high inpatient and early postdischarge mortality in patients hospitalised with $\mathrm{HF}$, it is surprising that there are very few data on the effectiveness of inpatient HF teams. McDonald et al examined the impact of an HF programme in a small randomised, controlled study of 70 patients admitted with HF. They demonstrated that a combined multidisciplinary care programme, starting as an inpatient, eliminated 1-month readmissions in a population with a $20 \%$ admission rate in the month before study commencement. In contrast to our study, patients in the routine care group and those in the multidisciplinary HF programme were all managed by cardiologists. The only difference between the groups was the education given to the multidisciplinary group. Both groups received similar medical therapy, and interestingly readmissions were also eliminated in the routine care group.

In a separate large $(n=1504)$ US randomised study, a regional, multihospital quality improvement intervention failed to improve the length of stay, mortality, readmissions or quality of life in patients admitted with HF. ${ }^{10}$ However, this study involved implementation of standardised management care pathways rather than providing direct specialist clinical input individualised for each patient.

Our findings therefore build on a limited literature and support direct involvement of specialist multidisciplinary HFTs, starting during hospital admission. Although it is not a randomised study, the findings are real-world data, and despite very similar baseline characteristics the outcome of the two groups was very different.

Given the observational nature of the data, it is not possible to establish what aspects of HFT care, if any, reduced patient mortality. However, it seems likely that specialist care is important. Currently there is no national standardised care pathway for patients admitted with HF in the UK. Some patients are managed by cardiologists (or other physicians) with a specialist interest and expertise in HF. In contrast others are admitted under cardiologists or physicians without such specialist expertise. Allocation of care to a given physician is often somewhat arbitrary despite evidence that outcomes are improved in patients managed by cardiologists. ${ }^{11}$ This is in stark contrast to the care given to patients with some other conditions with comparable mortality, for example, cancers where management by an oncologist is appropriately considered to be standard care. Specialist care is likely to be the reason why the post-HFT group was more likely to receive evidence-based drug therapy for HF, including ACE-I and MRAs which improve outcome in patients with severe HF. ${ }^{12} 13$ After the introduction of HFT, the prescribing rates of all prognostically important medications were comparable with rates achieved in the National Heart Failure Audit. ${ }^{1}$ In addition, post-HFT patients received more intensive diuretic therapy. Although these drugs have not been clearly shown to improve outcome, they are important symptomatically. Interestingly post-HFT patients were not more likely to receive beta-blockers or more advanced HF therapies. This suggests that most patients in whom inpatient beta-blocker therapy was appropriate were already receiving these drugs under non-specialist care. However, postdischarge initiation and up-titration of medications may have been more likely to occur with specialist follow-up. The similar rates of advanced and more intensive therapies may reflect the fact that these treatments are usually reserved for the most unwell patients and that these patients were more likely to be referred to and managed by cardiology before the HFT was set up. However, it is not possible to determine this from our data. Moreover, advanced device therapy is often not performed as an inpatient as most patients undergo a period of stabilisation and/or optimisation of medical therapy and reassessment before being put forward for device implantation.

An interesting observation was that post-HFT patients received more intensive diuretic therapy. The HFT goal was always to relieve congestion using whatever dose or combination of diuretics was necessary, whereas it is our experience that general physicians use diuretics more cautiously, particularly in the setting of renal dysfunction. Such patients treated by HFT received more intravenous and higher doses of loop diuretics and were more likely to be treated with a combination of thiazide and loop diuretics. Although furosemide was the most frequently prescribed loop diuretic on discharge, a greater proportion of post-HFT patients received bumetanide, which has a more predictable bioavailability. ${ }^{14}$ Diuretic dose has been shown to predict mortality in $\mathrm{HF}^{15}$; however, one study suggested that clinical stability may be more important than baseline diuretic dose. ${ }^{16}$ Reducing cardiac filling pressures in $\mathrm{HF}$ reduces sympathetic activation, ${ }^{17}$ and as such it is biologically plausible that using higher dose diuretics to achieve euvolaemia could improve outcome. Indeed freedom from congestion has been shown to predict survival in class IV patients. ${ }^{18}$

The multidisciplinary nature of the HFT is also likely to be an important factor with different healthcare professionals complimenting one another. HF nurses are at the 
centre of the HFT and are key to many aspects of care previously shown to improve outcome, including education and improved patient self-care, follow-up monitoring and access to specialised HF clinics. ${ }^{8}$

We did not observe a reduction in readmissions seen as a result of multidisciplinary team care in previous studies. ${ }^{8}$ This difference may be a result of our HFT focusing on inpatient care and the first 2 weeks following discharge; community HFTs were available to manage HF patients postdischarge in both cohorts. Also relevant is the fact that by markedly reducing inpatient mortality in our HFT cohort, many sicker patients survived to discharge. In this context maintaining the same level of HF readmissions could be considered a success.

Although much ongoing HF research is focused on complex imaging and drug and device therapies, it is encouraging to note the potential impact of an inpatient HFT on mortality. Our absolute risk reduction in mortality on discharge was $16 \%$ ( $6 \%$ vs $22 \%$ ), which is clinically very relevant. This was maintained at 1 year (27\% vs $43 \%)$. Although direct comparisons are not possible landmarks, $\mathrm{HF}$ trails have shown an absolute risk reduction in mortality of $18 \%$ with ACE-I, ${ }^{12} 11 \%$ with spironolactone, ${ }^{13} 5.5 \%$ with bisoprolol, ${ }^{19} 10 \%$ with Cardiac Resynchronisation Therapy (CRT) ${ }^{20}$ and $7 \%$ with Implantable Cardioverter Defibrillator (ICDs) ${ }^{21}$ It is important for policymakers to remember that HF is a chronic condition, and involvement of multidisciplinary teams provides patient-centred care and also improves outcomes and patient experience. Furthermore, in a condition that still has an unacceptably high inpatient mortality, early involvement of this team seems to be beneficial.

\section{LIMITATIONS}

These are observational data rather than a randomised controlled trial, and as such it is not possible to categorically separate causation and association. However, we believe it would be difficult ethically to design a conventional randomised controlled trial at this stage given the evidence that outcomes are improved by specialist input. ${ }^{1}$ Also, it is difficult to randomise patients within a hospital to differences in the level of service. Management almost inevitably improves in the control group, which may lead to a neutral outcome. Randomising centres to initiate or defer the creation of such a service in a cluster randomised trial might be possible and preferable. ${ }^{22}$

Not all patients admitted with HF were seen by the HFT as onward referral was required by the admitting team. Given that a similar number of patients were admitted with HF in the 6 months before the HFT was set up, this suggests that the HFT saw approximately half of all admissions in its first operational 12 months. Consequently we cannot exclude a referral bias in favour of the post-HFT cohort. However, the baseline characteristics suggest that the two populations were very similar, while accepting that no assessment of cognitive function was made which might adversely affect patient treatment and outcome. It is interesting to note that following successful discharge the subsequent mortality within the 1-year follow-up period was identical for both groups at $21 \%$, which further supports the belief that the two patient populations were well matched (pre-HFT inpatient mortality 22\%, 1-year mortality $43 \%$ vs post-HFT inpatient mortality $6 \%$, 1-year mortality 27\%). Thus, the impact of the HFT does appear to be real.

This report is predominantly focused on inpatient care; specialist follow-up was variable (cardiology, HFT, primary care HF nurse) and some patients were discharged from the service within a few months. Patients could have been hospitalised elsewhere during follow-up, but we would expect any underestimation in readmissions to be equally balanced between the two cohorts.

\section{CONCLUSIONS}

Specialist multidisciplinary team input for patients hospitalised with decompensated HF is associated with significantly reduced inpatient and 1-year mortality. Improved use of evidence-based drug therapies, together with more intensive diuretic use, and the multidisciplinary nature of the team may contribute to differences in patient outcome.

Contributors JM designed the study, analysed and interpreted the data and critically revised the manuscript. GM assisted with study design, analysed and interpreted the data and drafted the manuscript. IA, JS, EG, JG, ASF helped acquire and analyse the data and critically revised the manuscript. JGFC analysed the data and critically revised the manuscript. PJC designed the study protocol, analysed the data and drafted the manuscript. All authors read and approved the final manuscript. PJC is responsible for the overall content as guarantor.

Competing interests None declared.

Provenance and peer review Not commissioned; internally peer reviewed.

Data sharing statement No additional data available.

Open Access This is an Open Access article distributed in accordance with the Creative Commons Attribution Non Commercial (CC BY-NC 4.0) license, which permits others to distribute, remix, adapt, build upon this work non-commercially, and license their derivative works on different terms, provided the original work is properly cited and the use is non-commercial. See: http://creativecommons.org/ licenses/by-nc/4.0

(c) Article author(s) (or their employer(s) unless otherwise stated in the text of the article) 2017. All rights reserved. No commercial use is permitted unless otherwise expressly granted.

\section{REFERENCES}

1 National Heart Failure Audit report. 2013-2014. http://www.ucl.ac.uk/ nicor/audits/heartfailure/documents/annualreports/hfannual13-14. pdf (accessed 6 Oct 2016).

2. National Institute for Health and Care Excellence. 2010. CG108 chronic heart failure: management of chronic heart failure in adults in primary and secondary care. http://publications.nice.org.uk/chronicheart-failurecg108 (accessed 6 Oct 2016).

3. Cowie MR, Wood DA, Coats AJ, et al. Incidence and aetiology of heart failure; a population-based study. Eur Heart J 1999;20:421-8.

4. Davies M, Hobbs F, Davis R, et al. Prevalence of left-ventricular systolic dysfunction and heart failure in the echocardiographic Heart of England Screening study: a population based study. Lancet 2001;358:439-44.

5. Ponikowski $P$, Voors AA, Anker SD, et al. ESC guidelines for the diagnosis and treatment of acute and chronic heart failure: the task force for the diagnosis and treatment of acute and chronic heart failure of the European Society of Cardiology (ESC). Eur J Heart Fail 2016;18:891-975. 
6. Yancy CW, Jessup M, Bozkurt B, et a/Writing Committee Members,; American College of Cardiology Foundation/American Heart Association Task Force on Practice Guidelines. 2013 ACCF/ AHA Guideline for the management of heart failure: a report of the American College of Cardiology Foundation/American Heart Association Task Force on Practice Guidelines. Circulation 2013;128:1810-52.

7. Holland R, Battersby J, Harvey I, et al. Systematic review of multidisciplinary interventions in heart failure. Heart 2005;91:899-906

8. McAlister FA, Stewart S, Ferrua S, et al. Multidisciplinary strategies for the management of heart failure patients at high risk for admission: a systematic review of randomized trials. J Am Coll Cardiol 2004;44:810-9.

9. McDonald K, Ledwidge M, Cahill J, et al. Elimination of early rehospitalization in a randomized, controlled trial of multidisciplinary care in a high-risk, elderly heart failure population: the potential contributions of specialist care, clinical stability and optimal angiotensin-converting enzyme inhibitor dose at discharge. Eur $J$ Heart Fail 2001:3:209-15.

10. Philbin EF, Rocco TA, Lindenmuth NW, et al. The results of a randomized trial of a quality improvement intervention in the care of patients with heart failure. The MISCHF Study Investigators. Am J Med 2000;109:443-9.

11. Reis SE, Holubkov R, Edmundowicz D, et al. Treatment of patients admitted to the hospital with congestive heart failure: specialtyrelated disparities in practice patterns and outcomes. J Am Coll Cardiol 1997;30:733-8.

12. Anon A. Effects of enalapril on mortality in severe congestive heart failure. results of the cooperative north scandinavian enalapril survival study (CONSENSUS). The CONSENSUS Trial Study Group. N Engl J Med 1987;316:1429-35.
13. Pitt B, Zannad F, Remme WJ, et al. The effect of spironolactone on morbidity and mortality in patients with severe heart failure. Randomized Aldactone Evaluation Study Investigators. N Engl J Med 1999;341:709-17.

14. Wargo KA, Banta WM. A comprehensive review of the loop diuretics: should furosemide be first line? Ann Pharmacother 2009:43:1836-47.

15. Eshaghian S, Horwich TB, Fonarow GC. Relation of loop diuretic dose to mortality in advanced heart failure. Am J Cardiol 2006;97:1759-64.

16. Mielniczuk LM, Tsang SW, Desai AS, et al. The association between high-dose diuretics and clinical stability in ambulatory chronic heart failure patients. J Card Fail 2008;14:388-93.

17. Azevedo ER, Newton GE, Floras JS, et al. Reducing cardiac filling pressure lowers norepinephrine spillover in patients with chronic heart failure. Circulation 2000;101:2053-9.

18. Lucas C, Johnson W, Hamilton MA, et al. Freedom from congestion predicts good survival despite previous class IV symptoms of heart failure. Am Heart J 2000;140:840-7.

19. The cardiac insufficiency bisoprolol study II (CIBIS-II): a randomised trial. Lancet 1999;353:9-13.

20. Cleland JG, Daubert JC, Erdmann E, et al; Cardiac Resynchronization-Heart Failure (CARE-HF) Study Investigators. The effect of cardiac resynchronization on morbidity and mortality in heart failure. N Engl J Med 2005;352:1539-49.

21. Bardy GH, Lee KL, Mark DB, et al; Sudden Cardiac Death in Heart Failure Trial (SCD-HeFT) Investigators. Amiodarone or an implantable cardioverter-defibrillator for congestive heart failure. N Engl J Med 2005;352:225-37.

22. Hemming K, Haines TP, Chilton PJ, et al. The stepped wedge cluster randomised trial: rationale, design, analysis, and reporting. BMJ 2015;350:h391. 